\title{
The ViRVIG Institute
}

\author{
Carlos Andújar, Isabel Navazo, Pere-Pau Vázquez \\ MOVING Group \\ Universitat Politècnica de Catalunya \\ Emails: \{andujar|isabel|ppau\}@1si.upc.edu
}

\author{
Gustavo Patow, Xavier Pueyo \\ GGG \\ Universitat de Girona \\ Emails: \{patow|xavier\}@ima.udg.edu
}

\begin{abstract}
In this paper we present the ViRVIG Institute, a recently created institution that joins two well-known research groups: MOVING in Barcelona, and GGG in Girona. Our main research topics are Virtual Reality devices and interaction techniques, complex data models, realistic materials and lighting, geometry processing, and medical image visualization. We briefly introduce the history of both research groups and present some representative projects. Finally, we sketch our lines for future research.
\end{abstract}

Keywords: 3D User Interfaces; Virtual Reality

\section{INTRODUCTION}

The ViRVIG institute stands for "Visualització, Realitat Virtual i Interacció Gràfica", in Catalan, which means Visualization, Virtual Reality and Graphics Interaction. The institute was created at the beginning of year 2011, although the joint research group, with the same name, dates from 2009. The ViRVIG research group is the result of the federation of two pioneering computer graphics groups in Spain: the MOVING group from Universitat Politcnica de Catalunya, in Barcelona, and the GGG group from the Universitat de Girona.

The institute is placed in Catalonia, an autonomous region at the north-east of Spain. It has two locations, one in Girona, and the other one in Barcelona, and has been initially headed by professor Pere Brunet. The institute is formed by 17 faculty and $13 \mathrm{PhD}$ students. The federation of groups is relatively recent, and the groups are internationally known as separate groups. Therefore, we will present the groups separately.

\section{A. History}

The history of the lab is the history of the two research groups that have founded it. Since their creation, both groups have had a tight collaboration. There is, however some differences, since the fields of research are not exactly the same.

1) MOVING Group: The Modeling, Visualization, Interaction and Virtual Reality Group (MOVING) from Universitat Politècnica de Catalunya started its research activities back in 1980. At that time, the research lines focused mainly on geometric modeling. Throughout the years, the group expanded its research lines to other fields such as multiresolution, visualization in medicine, advanced user interfaces, virtual reality, and crowd simulation. The group started under the direction of Professor Pere Brunet. In 1984, the group started giving lectures on Computer Graphics. Around $35 \mathrm{PhD}$ students have completed their work since then.

The group has been one of the pioneering research groups in Computer Graphics in Spain, and has had a strong presence in international conferences and journals. They organized national and international conferences such as the EuroGraphics conference in 1993, the IEEE/EG Symposium on Data Visualization in 2002, the EG Workshop on Virtual Environments in 2002, or the EG Symposium on Geometry Processing in 2007. The researchers in the group have also participated in numerous Program Committees and has consolidated collaboration relationships with several researchers in universities and Research Centers across the world: Technical University of Vienna, Georgia Tech, Technical University of Darmstadt, Consiglio Nazionale delle Ricerche (CNR) in Pisa, MaxPlanck Institut in Saarbrücken, University of Pennsylvania, or Technical University of Eindhoven.

Additional information on the group can be found at http://www.lsi.upc.edu/ moving/.

2) GGG Group: The Geometry and Graphics Group (GGG) is a pioneer research group of the University of Girona. The team started its activities back in 1993, and has worked on rendering and computational geometry issues ever since. In these particular topics, the team has participated and headed several research projects in which their members have taken an active role (mainly as principal researchers).

The GGG has signed cooperation agreements with a number of companies in the areas of artificial and natural lighting, video games and software design, both at national and international level. Members of the team have participated in the creation of the EuroGraphics Working Group on Rendering and participated in the organization and chaired the main international workshops and conferences. They recently hosted the International Symposium on Rendering, one of the main activities in the international Computer Graphics field and will organize EuroGraphics 2013. Also, members of the team can be found at editorial boards of international journals as well as acting as reviewers of the main conferences and journals.

Team members had formed part of the executive committees of the Spanish and European Associations for Computer Graphics, and of the Workshops and Working Group Board of the European association and chaired it. The Geometry and Graphics Group frequently collaborates with national and international groups through internships, research stays, exchange of researchers and joint projects. We may underline those held with INRIA in Bordeaux, Rennes, Grenoble, and Nice, Université de Saint Etienne, Technical University of Budapest, Université de Montreal, University of Bristol, Carleton University in Ottawa (Canada), Denver University 
(USA), McGill University (Canada), and so on. The scientific contributions of the team members are regularly published in international journals and conferences.

Additional information on the group can be found in the web page: http://ggg.udg.edu/.

\section{B. Mission and goals}

The mission of the laboratory is the creation of a multidisciplinary research center where the scientific staff from UdG and UPC can develop joint research and technology transfer projects. Some of the concrete objectives are:

- Developing research in fields such as: Virtual Reality, advanced interaction, cooperative Virtual Reality systems, complex systems modeling, geometry processing, realistic rendering...

- The promotion of scientific relationships with other research centers, at a national and international level.

- To keep a tight link with the social and productive environment.

- To design and develop scientific educational programs in our fields of experience.

\section{Research lines}

Some of the most relevant research lines are:

- Virtual reality, immersive interaction and modelling.

- Visualization of complex n-dimensional information.

- Advanced 3D graphic interaction systems.

- Development of low cost Virtual Reality systems.

- Volume Rendering and Medical Illustrative Visualization.

- Architectural and urban design.

- Voronoi diagrams and partial matching problems.

- Mesh generation and processing.

- Realistic materials, rendering and lighting with complex light sources.

\section{Previous Projects}

Our group works in several projects in Computer Graphics, some of them involve the use and development of Virtual Reality tools and technologies.

\section{A. Development of Virtual Reality systems}

We conduct research on PC-based, immersive VR tables, portable VR systems, augmented reality systems, haptic interaction. The group has developed several low cost VR systems for specific applications and exhibitions. For these projects we obtained funding from UPC, private organizations, companies and the Spanish government.

Several years ago we developed the Mini-VR: A new virtual reality system designed to be small enough to be totally portable. It is a semi-immersive interaction system based on a movable stereoscopic projection screen with a tracking system to capture the movement of the screen with respect to the virtual model. The system can be used for cooperative inspection of very complex computer-aided design environments, allowing very simple interaction among users

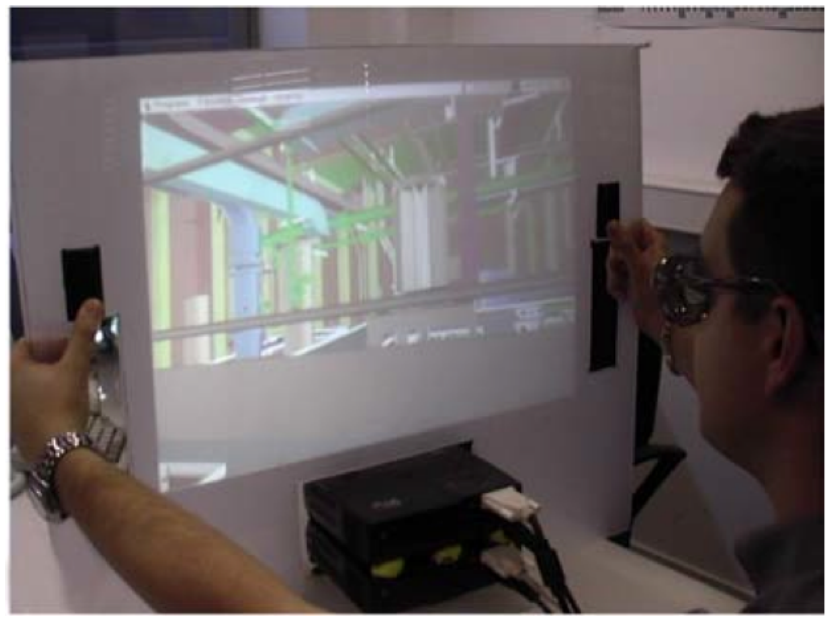

Fig. 1. The miniVR: a portable virtual reality device.

and designers at distant locations [1]. We can see this system in action in Figure 1.

One of our last projects consists on the development of a VR system with haptic and immersive performances, for neuron-surgical planning. This project has been funded by the Catalonia government. The project has been carried out in cooperation with Alma IT Systems and the Clinic Hospital in Barcelona.

\section{B. $3 D$ pointing and data inspection in $V R$ environments}

1) Enhanced Medical Data Inspection in a large VR setup: The simultaneous visualization of interior and exterior structures is complex in Volume Rendering. Often cut-away or exploded views are used. However, these methods require preprocessed data and accurate determination of the region of interest, previous to data inspection. Moreover, they are difficult to implement in realtime in VR environments.

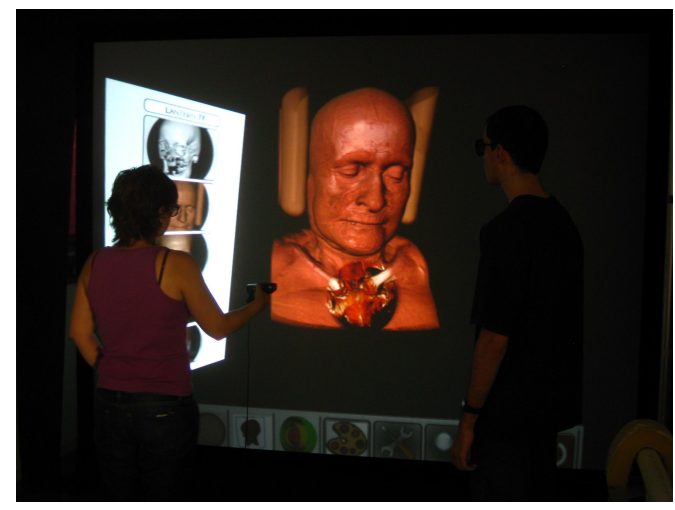

Fig. 2. Interaction session with our Virtual Magic Lantern

We have developed a new metaphor called the Virtual Magic Lantern [2] (see Figure 2). It behaves like a lantern whose virtual illumination cone provides the focal region which is visualized using a secondary transfer function or different 
rendering style. This may be used for simple visual inspection, surgery planning, or injure diagnosis. It is a particularly friendly and intuitive and our GPU-based implementation is able to run at realtime framerates with relatively complex models. It is suitable for environments with a large screen, where the user moves a Wanda device, like a lantern pointing to the model.

2) Improving $3 D$ pointing in Virtual Reality systems: Pointing is an important problem in VR environments, and it is required both for single user interactions and collaborative systems. Most pointing techniques for 3D selection in spatially immersive virtual environments rely on a ray originating at the user's hand and controlled by the hand orientation. However, mismatches between visible objects (those appearing unoccluded from the user's eye position) and selectable objects (those appearing unoccluded from the user's hand position) could hinder selection performance. We proposed a new device-ray mapping, Ray Casting from the Eye (RCE), to overcome this problem. In a controlled experiment involving selection tasks with complex 3D scenes, RCE clearly outperformed ray casting in terms of speed and accuracy [3].

Multi-user virtual reality systems enable natural interaction with shared virtual worlds. Users can talk to each other, gesture and point into the virtual scenery as if it were real. As in reality, referring to objects by pointing, results often in a situation whereon objects are occluded from the other users' viewpoints. Although this problem can only be solved by adapting the viewing position, specialized individual views of the shared virtual scene enable various other solutions.

We proposed show-through techniques to make sure that the objects one is pointing to can be seen by others [4] (see Figure 3 ). We analyzed the influence of such augmented viewing techniques on the spatial understanding of the scene, the speed of mutual information exchange as well as the social behavior of users. Our study revealed that show-through techniques support spatial understanding on a similar level as walking around to achieve a non-occluded view of specified objects. Moreover, they provide advantages in terms of comfort, user acceptance and compliance to social protocols, which suggest that VR techniques can in fact be better than 3D reality.

3) Illustrative Visualization of Medical Models: Volume models often show high depth complexity. This poses difficulties to the observer in judging the spatial relationships accurately. Illustrators usually use certain techniques such as improving the shading through shadows, halos, or edge darkening in order to enhance depth perception of certain structures. Both effects are difficult to generate in real-time for volumetric models because their high cost, or because they require precomputation that prevents changing the transfer function interactively, as it determines the occlusions. We have developed different illustrative visualization algorithms that simulate different effects such as ambient occlusion and halos both in 2D, that is, screen-space [5] or by taking into account the 3D environment of the point to lit [6]. We have also developed other rendering techniques to improve perception of depth and shapes of the models being inspected [7], [8]. In

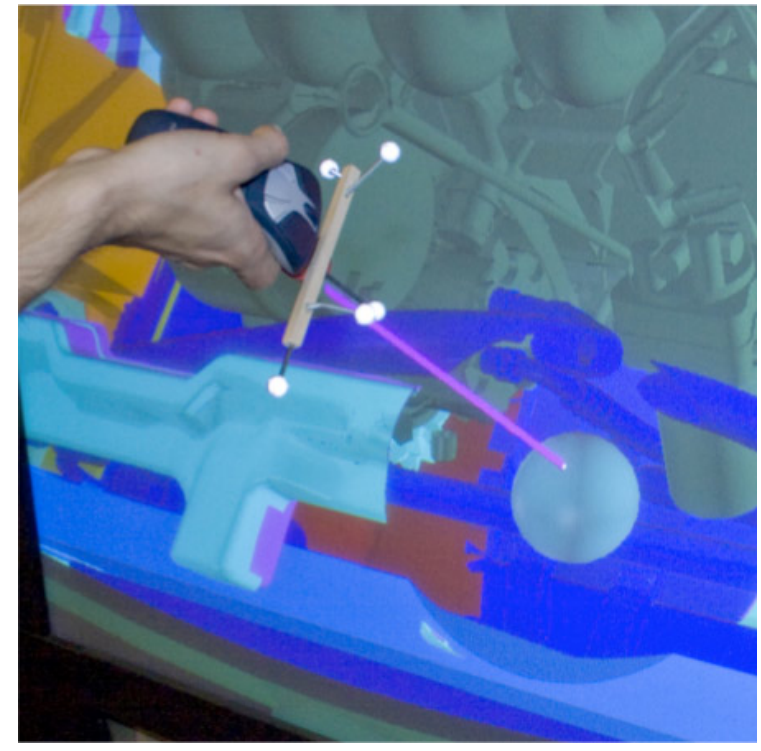

Fig. 3. Improved pointing in VR environments.

all cases, we take advantage of graphics hardware to accelerate our algorithms, either by using the normal OpenGL pipeline or by designing appropriate CUDA programs [6].

\section{Geometry and Texture Processing}

As a natural continuation of our initial work in Geometry Processing, we are focused on working with large and complex datasets, now also including textures, such as:

1) Multiscale Acquisition and Presentation of very large models: The dichotomy between full detail representation and the efficient management of data digitization is still a big issue in the context of the acquisition and visualization of 3D objects, especially in the field of the cultural heritage. Modern scanning devices enable acquiring very detailed geometry, but they also generate large artifacts. In this project we reconstructed the impressive $(7 \times 11 \mathrm{~m}$.) portal of the Ripoll Monastery, Spain. It was acquired using triangulation laser scanning, producing a dataset of $2 \mathrm{~K}$ range maps for a total of $1 \mathrm{~B}+$ triangles. We developed all the steps from the acquisition planning to the final setup for public dissemination [9]. Some of the most challenging parts of the project are imperfection repairing, and an interactive and immersive rendering in a publicly placed (MNAC museum in Barcelona) setup of a fully detailed representation of the portal (see Figure 4).

2) Inverse Geometry Textures: Inverse geometric textures (IGT) introduces a novel random-access parallel data-structure for interactive rendering [10]. The key idea is to map surface and color input data to a virtual regular grid, which naturally suits for the graphics hardware. This technique decouples surface and textures from high resolution meshes and map them onto lower resolution simpler domains, which are faster to render but still preserve the original details (see Figure 5).

3) Continuity Mapping of Multi-chart Textures: Continuity mapping can make any multi-chart parameterization to 


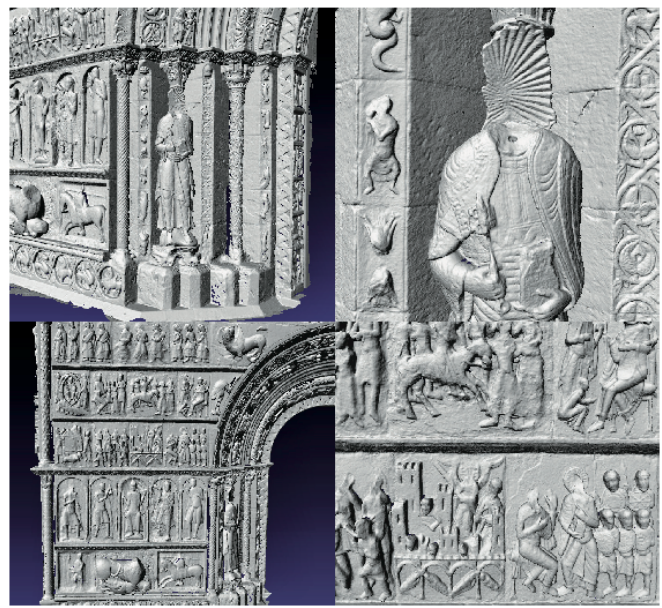

Fig. 4. Snapshots of the geometry of the 3D model obtained. Note the variety of the decorative elements and, in close-up, their intricate details.
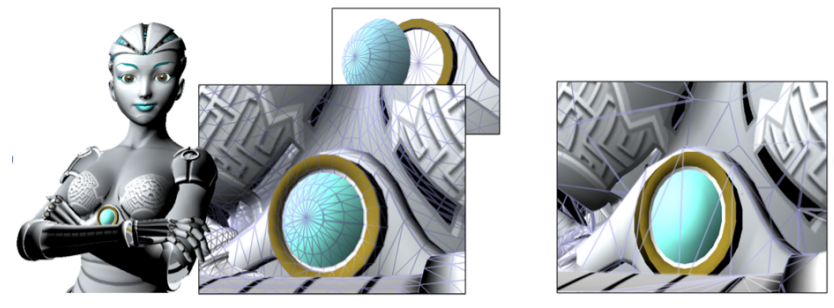

Fig. 5. Inverse Geometric Textures

be seamless, without requiring re-parameterizing the artistprovided textures, or non-accurate texturing operations like texture transfers (see Figure 6), with small memory and computing costs [11].

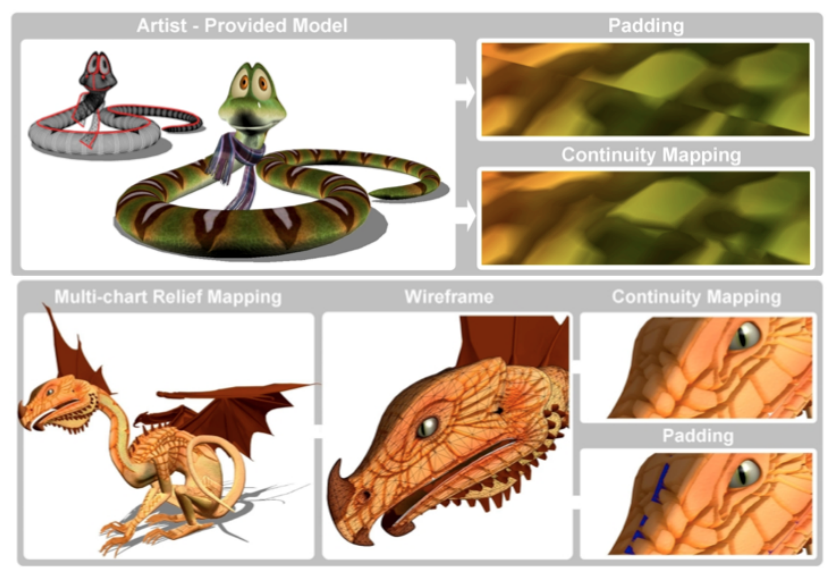

Fig. 6. Continuity Mapping of Multi-chart textures

4) skylineEngine: An urban procedural modeling system: skylineEngine is an urban procedural modeling tool developed as a test-bed for new algorithms and techniques in urban modeling. It has many features only available on high-end commercial modeling systems, as well as some novel features that make it a unique system, like a graph-based paradigm that allows the user to create content rich cities with distinct districts, major and minor roads, blocks, lots and buildings, and also other urban elements like streets, sidewalks, parks, bridges and landmarks [12].

5) Voronoi diagrams: The Voronoi diagram of a set of objects in a space is the partition of this space in regions in such a way that points of the same region have the same objects as the nearest or farthest objects. We are interested in computing approximated Voronoi diagrams in the plane, in the $3 \mathrm{D}$ space or on terrains. In the last case, we study how to properly compute distances on terrains [13].

\section{Current Projects And Potential COLlabORATIONS}

We are carrying out several projects such as:

- Virtual Reality advances for cutting-edge applications such as airplane design.

- Development of affordable Virtual Reality environments.

- HDR Imaging for uncontrollable environments.

- Digital capture, storage transmission, and display or realworld lighting.

- Visualization of medical datasets in mobile devices.

Thus, we are open to international collaborations in these areas of research. We have had many international $\mathrm{PhD}$ students and visitors for many years. We encourage interested enthusiastic students to come and work with us.

\section{REFERENCES}

[1] M. F. González, P. Brunet, and T. Techmann, "Minivr: a portable virtual reality system," Computers \& Graphics, vol. 28, no. 2, pp. 289-296, 2004.

[2] E. Monclús, J. Díaz, I. Navazo, and P.-P. Vázquez, "The virtual magic lantern: an interaction metaphor for enhanced medical data inspection," in Proceedings of the 16th ACM Symposium on Virtual Reality Software and Technology, 2009, pp. 119-122.

[3] F. Argelaguet and C. Andujar, "Efficient 3d pointing selection in cluttered virtual environments," Computer Graphics and Applications, vol. 29, pp. 34-43, 2009.

[4] F. Argelaguet, A. Kunert, A. Kulik, and B. Froehlich, "Improving colocated collaboration with show-through techniques," 2010, pp. 55-92.

[5] J. Díaz, H. Yela, and P.-P. Vázquez, "Vicinity occlusion maps: Enhanced depth perception of volumetric models," in Computer Graphics International, 2008, pp. 56-63.

[6] J. Díaz, P.-P. Vázquez, I. Navazo, and F. Duguet, "Technical section: Real-time ambient occlusion and halos with summed area tables," Comput. Graph., vol. 34, pp. 337-350, August 2010.

[7] J. Díaz and P.-P. Vázquez, "Depth-enhanced maximum intensity projection," in Volume Graphics, 2010, pp. 93-100.

[8] J. Díaz, J. Marco, and P.-P. Vázquez, "Cost-effective feature enhancement for volume datasets," in $V M V, 2010$, pp. 187-194.

[9] M. Callieri, A. Chica, M. Dellepiane, I. Besora, M. Corsini, J. Moyés, G. Ranzuglia, R. Scopigno, and P. Brunet, "Multiscale acquisition and presentation of very large artifacts: The case of portalada," J. Comput. Cult. Herit., vol. 3, pp. 14:1-14:20, April 2011.

[10] I. Garcia and G. Patow, "Igt: inverse geometric textures," ACM Trans. Graph., vol. 27, no. 5, pp. 1-9, 2008.

[11] F. González and G. Patow, "Continuity mapping for multi-chart textures," ACM Trans. Graph., vol. 28, pp. 109:1-109:8, December 2009.

[12] G. Patow, "User-friendly graph editing for procedural buildings," IEEE Computer Graphics and Applications, vol. 99, no. PrePrints, 2010.

[13] M. Fort and J. A. Sellarès, "Computing generalized higher-order voronoi diagrams on triangulated surfaces," Applied Mathematics and Computation, vol. 215, no. 1, pp. 235-250, 2009. 